\title{
Rheology of wet granular materials in shear flow: experiments and discrete simulations
}

\author{
Michel Badetti ${ }^{1}$, Abdoulaye Fall ${ }^{1}$, Jean-Noël Roux ${ }^{1 \mathrm{a}}$ \\ ${ }^{1}$ Laboratoire Navier, 2 Allée Kepler, 77420, Champs-sur-Marne, France
}

\begin{abstract}
The behaviour of wet granular media in shear flow is characterized by the dependence of apparent friction $\mu^{*}$ and solid fraction $\Phi_{S}$ on the reduced pressure $P^{*}$ and the inertia number $I$. Reduced pressure, $P^{*}=\sigma_{22} a^{2} / F_{0}$, compares the applied normal stress $\sigma_{22}$ on grains of diameter $a$ to the tensile strength of contact $F_{0}$ (proportional to the surface tension $\Gamma$ of the liquid and the beads diameter). A specifically modified rotational rheometer is used to characterize the response of model wet granular material (beads with diameter of submillimetric range) to applied shear rate $\gamma^{\prime}$ under controlled normal stress $\sigma_{22}$. Discrete Element Method (DEM) simulations in 3D are carried out in parallel and numerical results are compared with experimental ones. As $P^{*}$ is reduced, an increase of the apparent friction coefficient $\mu^{*}=\sigma_{12} / \sigma_{22}$, measured in the critical state and in slow flows with inertial effects is observed. While the agreement between experiments and simulations is good for dry materials as well as for wet materials in the quasistatic limit $(I \rightarrow 0)$, some differences appear at finite $I$, for which some possible origins are discussed.
\end{abstract}

\section{Introduction}

In their steady state, shear flows of granular materials under controlled normal stress $\sigma_{22}$ are well described by the solid fraction $\Phi_{S}$ and the apparent friction $\mu^{*}$ of the grains defined as:

$$
\begin{gathered}
\mu^{*}=\frac{\left\langle\sigma_{12}\right\rangle}{\sigma_{22}} \\
\Phi_{S}=\frac{\Omega_{S}}{\Omega}
\end{gathered}
$$

where $<\sigma_{12}>$ denotes the time average of the shear stress, $\sigma_{22}$ the normal confinement stress, $\Omega$ the sample volume and $\Omega_{S}$ the volume of the grains.

Flows of dry granular materials are commonly characterized $[1,2]$ by the inertial number $I$, defined as:

$$
I=\dot{\gamma} a \sqrt{\frac{\rho}{\sigma_{22}}}
$$

where $\dot{\gamma}$ is the shear rate, $a$ is the grain diameter and $\rho$ its mass density (e.g. $1050 \mathrm{~kg} . \mathrm{m}^{-3}$ for polystyrene). In the frequent case when elastic contact deflections are negligible, $I$ fully characterizes the state of the material in steady homogeneous shear flow. Constitutive laws express $\Phi_{S}$ and $\mu^{*}$ as functions of $I$ alone. Fig. 1 shows how $\mu^{*}$ and $\Phi_{S}$ vary throughout the flow regimes [1], in the quasi-static limit $\left(I<10^{-3}\right) \mu^{*}$ and $\Phi_{S}$ are constant. In the inertial regime $\left(10^{-3}<I<10^{-1}\right) \mu^{*}$ increases and $\Phi_{S}$

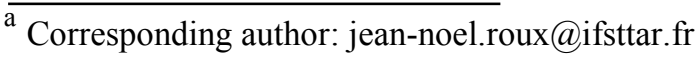

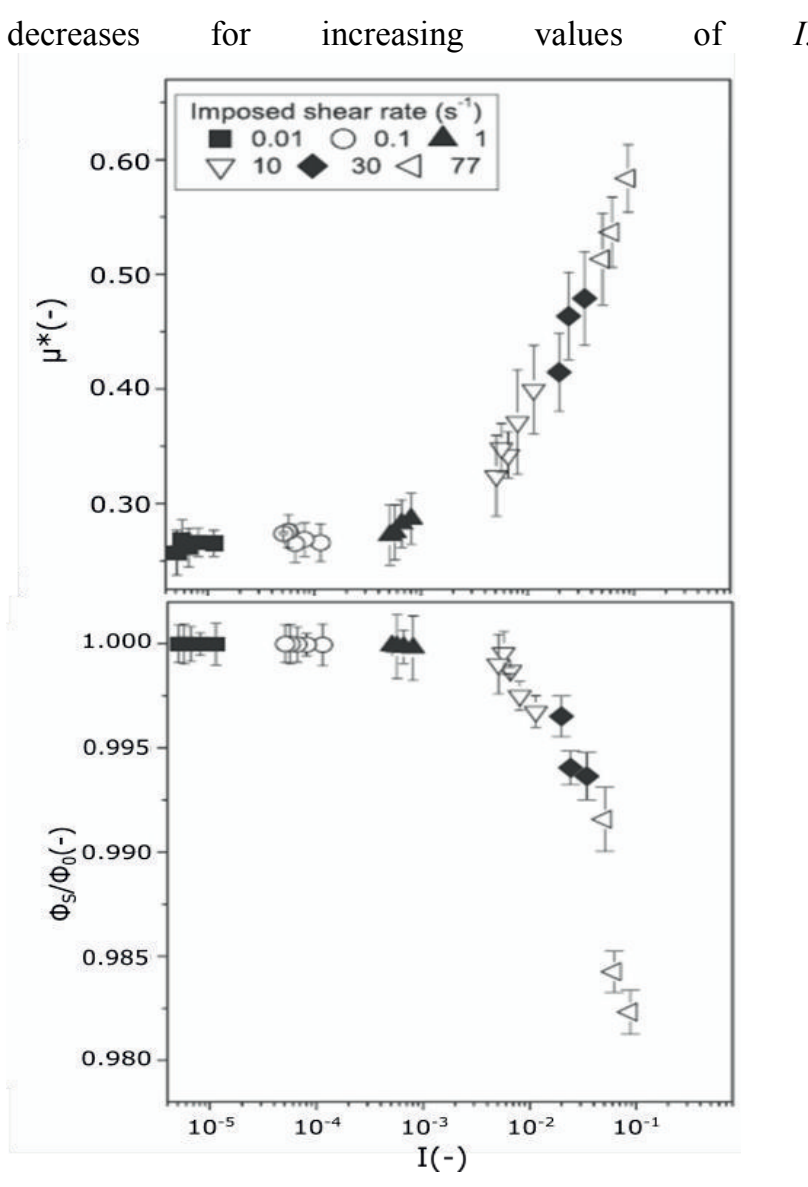

Figure 1: flow curve from [1], $\mu^{*}$ and $\Phi_{S} / \Phi_{0}$ versus $I\left(\Phi_{0}\right.$ being the solid fraction in the quasistatic limit). Notice the plateau for small values of $I$ corresponding to the quasistatic limit. 
In a cohesive material, intergranular contacts exhibit a tensile strength $F_{0}$ and the description of granular flow requires an additional dimensionless parameter, the reduced pressure $P^{*}$, which compares the cohesive effect with the normal stress :

$$
P^{*}=\frac{\sigma_{22} a^{2}}{F_{0}}
$$

Previous DEM studies [3] proposed constitutive laws in the form of $\Phi_{S}$ and $\mu^{*}$ as functions of both $I$ and $P^{*}$. Despite some investigations of the quasistatic limit [4] there are very few experimental studies of wet granular flow.

The aim of the present communication is to compare DEM simulations of wet grain assemblies to experimental rheometry using a normal stress controlled shear cell, both in the quasistatic limit and in dense inertial flows.

In section 2 the numerical model is presented, a particular focus is made on the cohesive force expression. Section 3 presents the experiments with descriptions of the model wet granular sample, the shear cell and the measurement procedure. In section 4, experimental and numerical results are compared. Further developments are discussed in section 5, stressing the potential interest of X-ray microtomographic investigations of the microstructure of sheared wet granular media.

\section{Numerical method}

\subsection{Principle}

Simple plane shear of a 3D box filled with $N$ spheres (4000 or 8800 ) of diameter $a$ is simulated. The box is periodic in all three directions $\mathbf{x}_{1}, \mathbf{x}_{2}, \mathbf{x}_{3}$. Fixed values of lengths $L_{1}=L_{3}$ are imposed and $\sigma_{22}$ is kept constant using a retroaction on length $L_{2}$. The shear originates from Lees-Edwards boundary condition, imposing a small displacement $\Delta / 2$ and $-\Delta / 2$ respectively to the top and bottom cell as illustrated on figure 2 . For homogeneous plane shear, shear deformation $\gamma$ is:

$$
\gamma=\frac{\Delta}{L_{2}}
$$

Contact and capillary forces are modelled as in $[2,3]$.

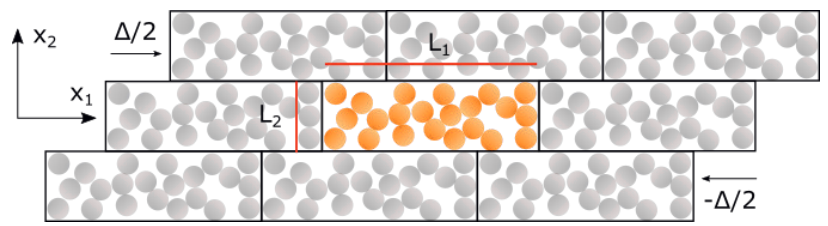

Figure 2 : Boundary conditions of Lees-Edwards. Grains in the main box (colored) are shifted along direction $\mathbf{x}_{1}$.

\subsection{Intergranular forces}

Contact forces are implemented following a simplified Hertz-Mindlin-Deresiewicz force model as in $[3,5]$. The friction in contact satisfies Coulomb's law with a friction coefficient $\mu=0.09$. This coefficient is a parameter that has been adjusted to fit the dry behaviour of our material in the quasistatic limit (details are given in section 4).

Working within the limits of the pendular state where the saturation degree $S_{w}$ lies roughly between $1 \%$ and $10 \%$, only simple liquid bridges between beads are present (see figure 3). Assuming a perfectly wetting fluid, the cohesive force $F_{c a p}$ in a bridge is approximated with the Maugis formula [3,6]:

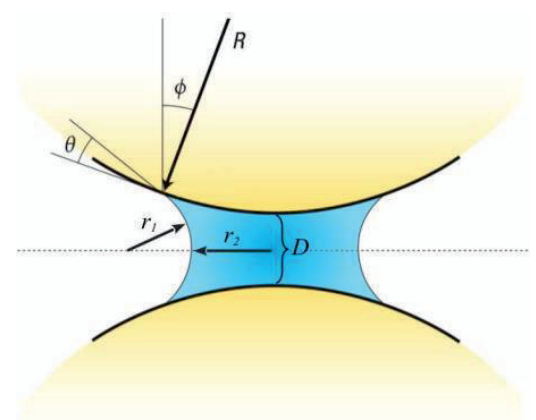

Figure 3: Simple cohesive bridge.

$$
F_{c a p}(d)=\pi \Gamma \mathrm{a}\left[1-\frac{1}{\sqrt{1+\frac{4 V}{\pi a d^{2}}}}\right]
$$

where $d$ denotes the distance between two spheres, $a$ the beads diameter, $\Gamma$ the surface tension and $V$ is the meniscus volume. Liquid bridges are assumed to form only once beads are in contact, however, the bridge breaks once a certain rupture distance $D_{\text {rupt }}$ is reached. A good approximation of this rupture distance is given by $[3,6]$ :

$$
D_{\text {rupt }}=V^{1 / 3}
$$

For negative values of $d$ (interpenetration) $F_{0}$ is assumed to be a constant equal to $F_{c a p}(0)$. Most parameters discussed above can be chosen according to the experimental material (see section 3.1). Setting $V / a^{3}=$ $10^{-3}$ corresponds to $\mathrm{S}_{\mathrm{w}}$ about $1 \%$.

\subsection{Numerical measurement}

Simulation runs are performed until a steady state is reached ( $\mu^{*}$ and $\Phi_{S}$ are constant $), \gamma$ is roughly a few units. In homogeneous plane shear the velocity $v$ is linear with coordinate $x_{2}$, a plot of these parameters is proposed on figure 4 , the linearity of $v$ with $x_{2}$ ensures a good measurement. 


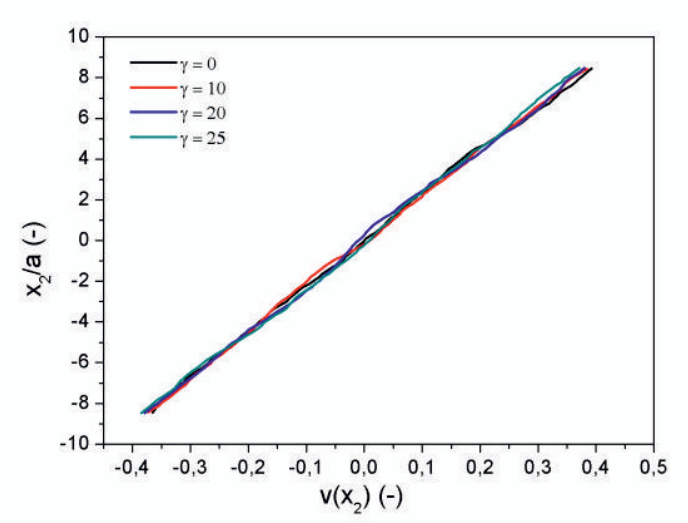

Figure 4: velocity profiles $v$ versus $x_{2}$, for different values of $\gamma$ obtained with $I=10^{-1}, P^{*}=1, N=4000$ and $V=10^{-3}$.

Values of $\Phi_{S}$ and $\sigma_{i j}$ are determined as in [3]. Averages and error bars are calculated using the method of block transform estimates [8] in steady state only. Figure 5 presents time series of normal and shear stresses and solid fraction in the steady state.
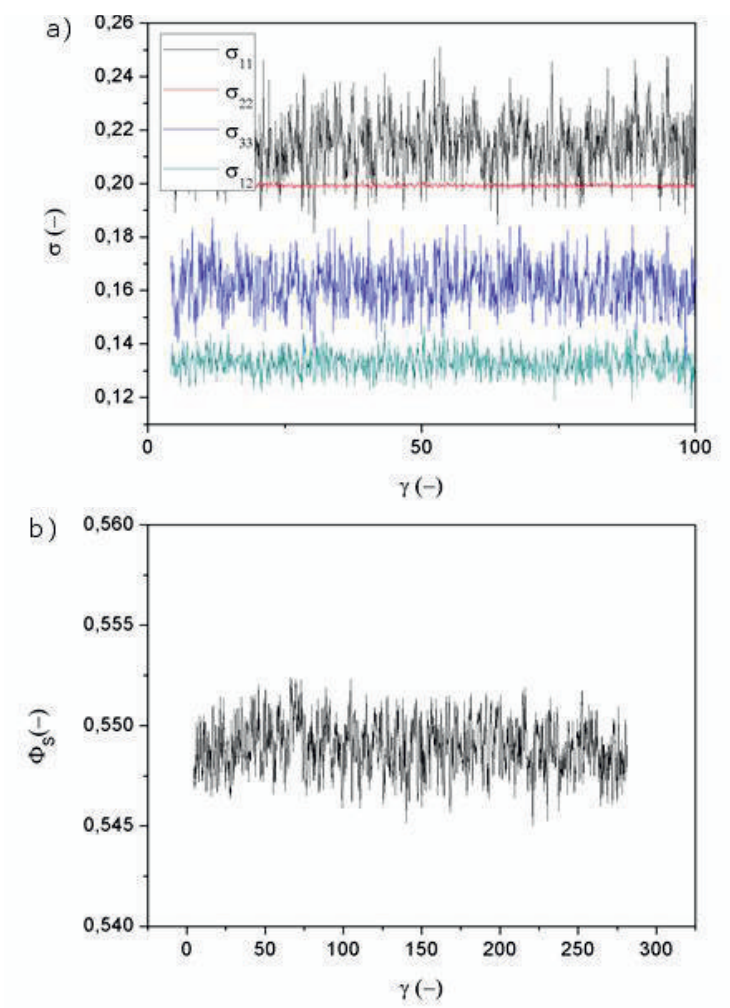

Figure 5: a) Normal stresses and shear stress, b) solid fraction versus $\gamma$. Time series obtained with $I=10^{-1}, P^{*}=1, N=4000$ and $V=10^{-3} \mathrm{a}^{3}$.

\section{Macroscopic experiments}

\subsection{Materials and samples}

The model material used is an assembly of dry polystyrene beads of diameter $a=500 \mu \mathrm{m}$ mixed together with a non-volatile, wetting, Newtonian silicon oil with a surface tension $\Gamma=47 \mathrm{mN} \cdot \mathrm{m}^{-2}$ and viscosity $\eta_{L}=$
$500 \mathrm{mPa}$.s. In practice, silicon oils almost perfectly wets polystyrene. The mass ratio between the oil and beads $m_{L} / m_{S}$ is controlled by weighing the ingredients before they are mixed. Knowing this ratio enables the control of the liquid saturation $S_{w}$ as long as the sample volume and mass are known.

\subsection{Rheological measurements}

Experimental devices must allow the control of the normal stress $\sigma_{22}$ and the shear rate $\dot{\gamma}$ and enable the measure of the shear stress $\sigma_{12}$. To this extent, an annular shear cell is used (see figure 6), it is assumed to produce a simple plane shear in the $\mathbf{x}_{1}, \mathbf{x}_{2}$ plane, similar to a planeplane geometry truncated in the center. Defining the inner $R_{i}$ and outer $R_{e}$ radii, the sample height $H$, the rotational velocity $\omega$, the normal force $F_{n}$ and the torque $T$, the averages of $\sigma_{12}, \sigma_{22}$ and $\dot{\gamma}$ can be expressed as:

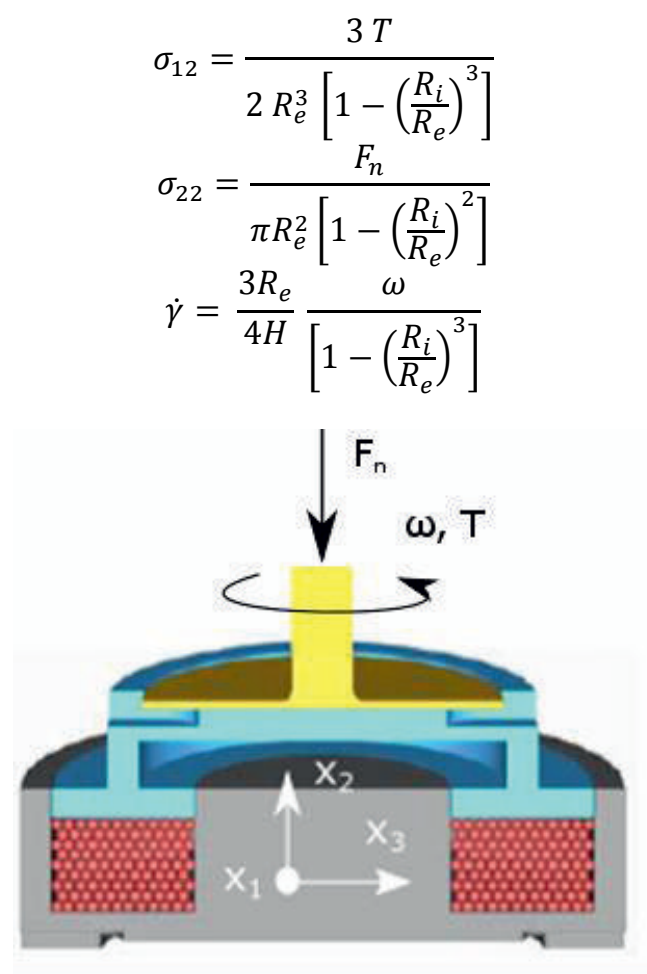

Figure 6: Experimental shear cell. The rotor (yellow) is bound to the top (blue), this ensemble can exert a pressure and a shear on the beads (red) which are confined in the base (grey). Perfect slipping is assumed on the sides (smooth polyoxymethylene), perfect sticking is assumed on top and bottom (square based polystyrene pyramids of $500 \mu \mathrm{m}$ in height and side).

In this study, 2 annular cells are used; they differ only by inner and outer radii:

- $\mathrm{A} 1-R_{i}=2.1 \mathrm{~cm}, R_{e}=4.5 \mathrm{~cm}$

- $\mathrm{A} 2-R_{i}=3.8 \mathrm{~cm}, R_{e}=6.2 \mathrm{~cm}$

Edge effects, such as local ordering around the top and bottom (see figure 6) and imperfect slip or loosening along the sides, are assumed negligible. Note that with imperfect slip, a dependence of $\mu^{*}$ on $H$ should appear. Results on dry materials [1] do not exhibit such dependence. The friction at the wall $\mu_{w}=0.05$ [1] is also 
small compared with the values of $\mu^{*}$ found experimentally (see section 4).

The order of magnitude of the normal stress in the experiment is $1 \mathrm{kPa}$. Considering the Young modulus of polystyrene beads, the stiffness modulus is well above $10^{4}$. With such order of magnitude of stiffness, it is reasonable to assume that beads behave as perfectly stiff.

Measurements are performed in the steady state, each sample is tested 6 times. For one material there are at least 2 samples used. Averages are deduced from all these measures, error bars are taken as the standard deviation from the averages calculated that way. Raw data from a measurement on a dry material are presented on figure 7. As well as simulations, the measures are performed on a $\gamma$ interval of several units at least.

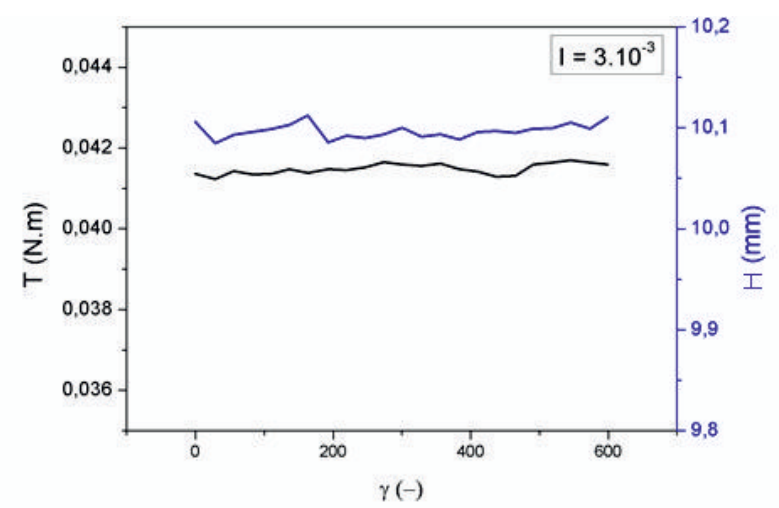

Figure 7: Torque (left axis, black curve) and cell height (right axis, blue curve) versus shear strain. Shear cell A1 is used with a pressure of $900 \mathrm{~Pa}$.

\section{Results}

Figure 8 shows both the numerical and the experimental results obtained for flow curves of dry granular materials, i.e. $\mu^{*}$ and $\Phi_{S}$ versus $I$. Note that the value of the intergranular friction coefficient $\mu$ used in the numerical model is chosen such that $\mu^{*}=0.25$ in the quasistatic limit, which is close to the values obtained for dry grains in experiments. This choice is comforted by the very close experimental and numerical values of $\Phi_{S}$ in this flow regime.

For dry materials, the agreement between the 2 cells (A1 and A2) and the simulations is very good. Differences are at most $4 \%$ for $\mu^{*}$ and $0.7 \%$ for $\Phi_{S}$ between $\mathrm{A} 1$ and $\mathrm{A} 2,7 \%$ and $0.7 \%$ between $\mathrm{A} 1$ and simulations, $10 \%$ and $0.3 \%$ between $\mathrm{A} 2$ and simulations. The good agreement between cells $\mathrm{A} 1$ and $\mathrm{A} 2$ indicates that assuming a constant shear stress is reasonable. The good agreement between experiments and simulations indicates that the experimental device is adequate to measure our material properties.

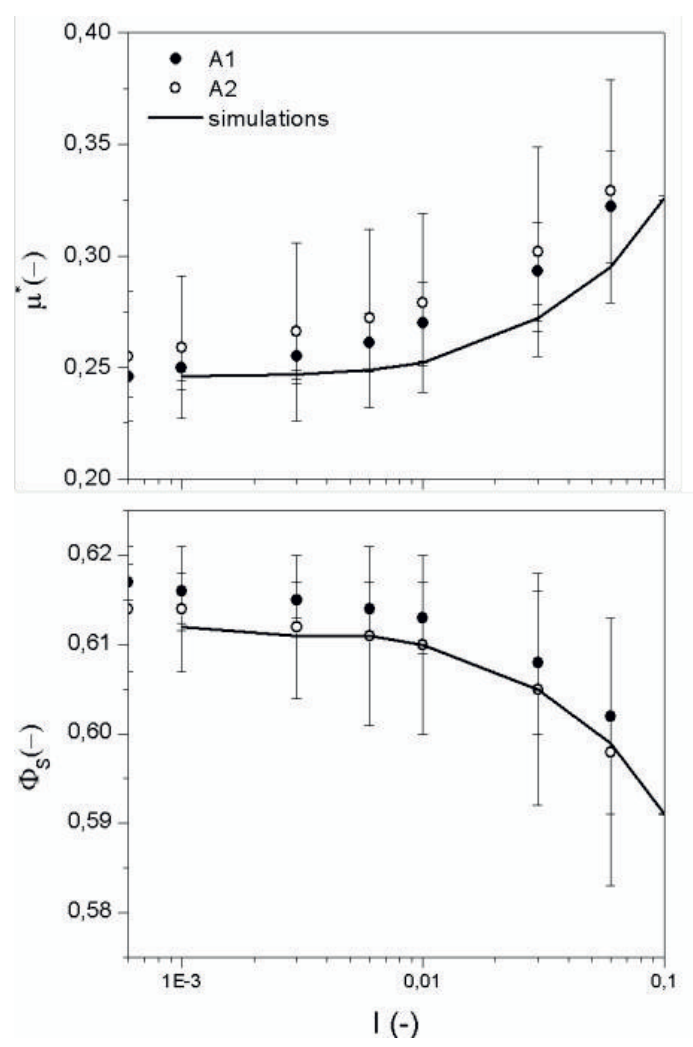

Figure 8: Flow curves of dry grains. Open and closed circles are experiments, solid line are numerical results.

Using now the A1 shear cell, friction coefficient and solid fraction, depending on $P^{*}$ and $I$ are shown and compared to numerical results on figure 9 . The agreement in the quasistatic limit seems good, with $I=10^{-3}$ :

- For $P^{*}=2-\mu^{*}=0.340$ and $\Phi_{S}=0.595$ in experiments versus $\mu^{*}=0.364$ and $\Phi_{S}=0.597$ in simulations.

- For $P^{*}=3-\mu^{*}=0.299$ and $\Phi_{S}=0.606$ in experiments versus $\mu^{*}=0.294$ and $\Phi_{S}=0.605$ in simulations.

As remarked in [3], in the quasistatic limit the increase of $\mu^{*}$ for decreasing $P^{*}$ is well predicted with an effective pressure argument, i.e. assuming the wet grains assembly behaves as a dry material with an additional pressure equal to the capillary pressure.

The agreement between simulations and experiments remains good when comparing the solid fractions for dense flows, the maximal relative difference between experiments and simulations amounts to $0.5 \%$ for $P^{*}=2$ and $0.8 \%$ for $P^{*}=3$. There is, however, a notable discrepancy when looking at the values of $\mu^{*}$ in the inertial regime. For higher values of I (above $3.10^{-3}$ ), the relative difference rises up to $30 \%$ for $P^{*}=3$ and up to $17 \%$ for $P^{*}=2$. In fact, it is particularly clear that $\mu^{*}$ increases much faster with $I$ in the experiments than in the numerical simulations. 


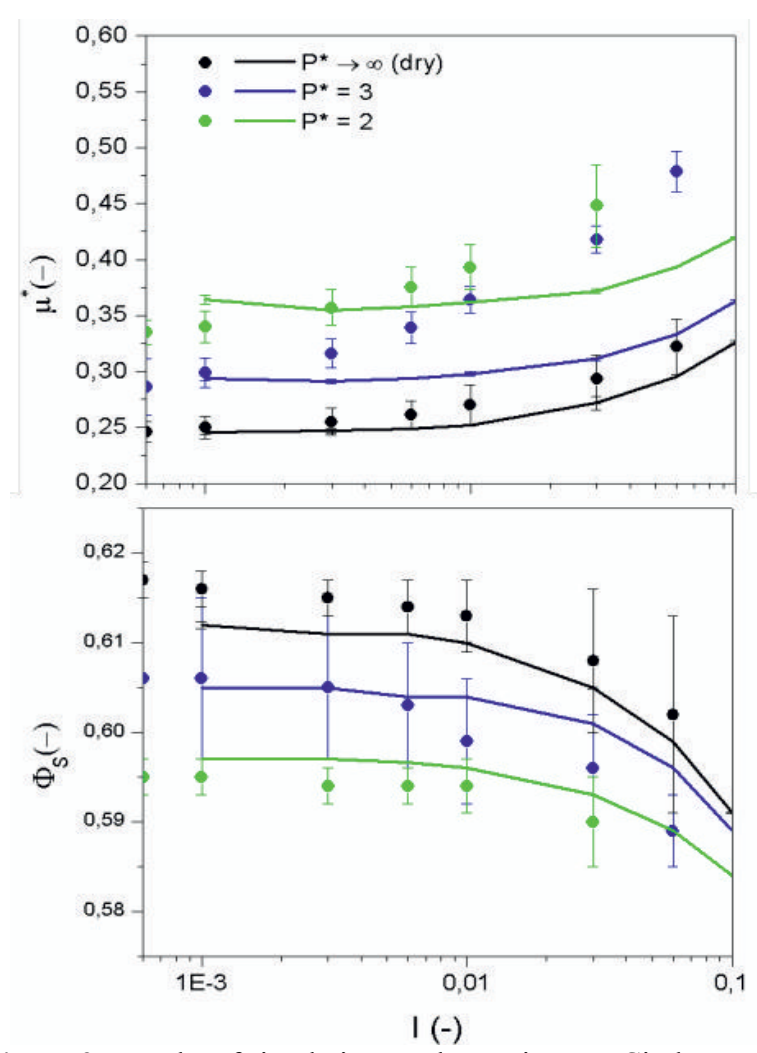

Figure 9: Results of simulations and experiments. Circles are experimental values, plain lines represent numerical values.

Although we do not have an explanation yet, possible clues are provided by the following 4 remarks.

- The fluid viscosity may impact the behaviour of the packing. With increasing viscosity, liquid bridges should take either a stronger force or longer time to break [9] giving rise to a more frictional packing.

- Heterogeneization (drainage, local ordering) may occur during experimental measurements. It is unclear how such occurrence would modify the macroscopic results obtained experimentally.

- Shear localization may occur during experiments [2]. In the case of simple shear, shear bands in the $\mathbf{x}_{\mathbf{1}}, \mathbf{x}_{\mathbf{3}}$ plane can be expected. Such localization would lead to an experimentally underestimated shear rate $\dot{\gamma}$, i.e. an underestimated $I$, which is consistent with the faster increase of $\mu^{*}$ with I found in experiments.

- Some menisci may exhibit a complex morphology despite working with small values of liquid saturation $\left(S_{w}\right.$ about 5\%) [10]. Characterizing the cohesive effect in such case is much less trivial as the Laplacian pressures can differ greatly from one morphology to another.

\section{Further developments}

New experimental samples, using different oil viscosity, as well as a thorough study of an eventual gap (defined in section 3.2) effect should give enough clues to assess whether the fluid viscosity or localization have an effect on the experimental measures.

X-ray microtomography experiments are also designed to help answer the questions raised in section 4 . They are performed on the samples described in section
3.1. 3D images are taken at different deformation steps. The images are composed of successive slices of materials in the plane perpendicular to the rotation axis (see Fig. 10).

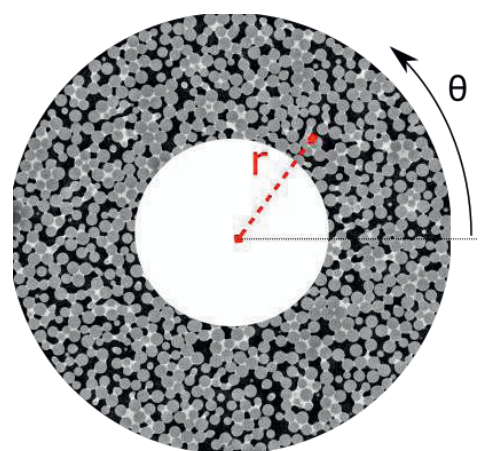

Figure 10: Microtomographic image of sample cut orthogonally to the axis of rotation.

Figure 11 is an example of $2 \mathrm{D}$ plot of the liquid saturation and solid fraction in the azimuthal plane $(\mathrm{r}, \mathrm{z})$, obtained by averaging over $\theta$ from 0 to $2 \pi$ (see Fig. 10). These pictures show evidence of local ordering and drainage (note the dryer region marked "-“ in the top part of the sample).

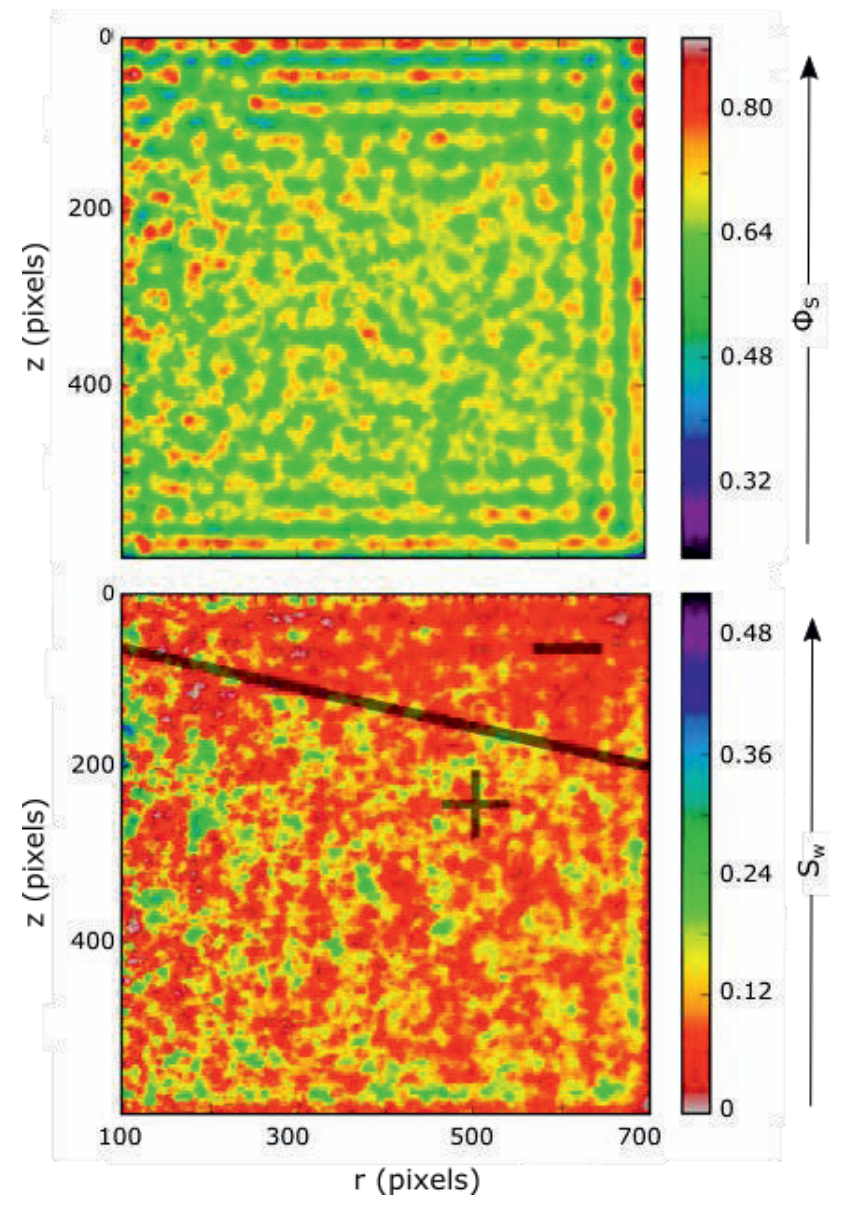

Figure 11: Map of solid fraction (top) and liquid saturation (bottom, global average is $10 \%$ here) as averaged over $\theta$, in (r, $\mathrm{z})$ plane (z oriented downwards). 


\section{References}

1. A. Fall, G. Ovarlez, D. Hautemayou, C. Mézière, J.N Roux, F. Chevoir, J. of Rheol., 59,4,(2015)

2. B. Andreotti, Y. Forterre, O. Pouliquen, Physics, (EDP Science, 2011)

3. S. Khamseh, J.-N Roux, F. Chevoir, Phys. Rev. E 92, 022201, (2015)

4. V. Richefeu, M. S. El Youssoufi, F. Radjaï, Phys. Rev. 73, 051304, (2006)

5. I. Agnolin, J.-N Roux, Phys. Rev. E 76, 061302, (2007)

6. O. Pitois, P. Moucheront, X. Chateau, J. Coll. Interf. Sci., 231, 26-31, (2000)

7. I. Agnolin, J.-N Roux, Phys. Rev. E 76, 061302, (2007)

8. H. Flyvbjerg, H. G. Petersen, J. Chem. Phys., 91, 1, (1989)

9. G. Lefebvre, P. Jop, Phys. Rev E, 88, 032205, (2013)

10. M. Scheel, R. Seemann, M. Brinkmann, M. Di Michiel, A. Sheppard, S. Herminghaus, Jour. Of Phys., 20, 494236, (2008) 